\title{
THE
}

$1-15-1996$

\section{Optical Power Limiting in Multilayer Systems with Nonlinear Response}

Leonard M. Kahn

University of Rhode Island, lenkahn@uri.edu

Follow this and additional works at: https://digitalcommons.uri.edu/phys_facpubs

Terms of Use

All rights reserved under copyright.

\section{Citation/Publisher Attribution}

Kahn, L. M. (1995). Optical power limiting in multilayer systems with nonlinear response. Physical Review B, 53(3), 1429-1437. doi: 10.1103/PhysRevB.53.1429

Available at: http://dx.doi.org/10.1103/PhysRevB.53.1429

This Article is brought to you for free and open access by the Physics at DigitalCommons@URI. It has been accepted for inclusion in Physics Faculty Publications by an authorized administrator of DigitalCommons@URI. For more information, please contact digitalcommons-group@uri.edu. 


\title{
Optical power limiting in multilayer systems with nonlinear response
}

\author{
Leonard M. Kahn \\ Department of Physics, University of Rhode Island, Kingston, Rhode Island 02881
}

(Received 25 April 1995)

\begin{abstract}
Optical power limiting is studied in multilayer systems where alternate layers exhibit nonlinear response. It is shown that the existence of optical limiting depends on the sign of the coupling of the Kerr nonlinearity. Approximate analytic results are found for single-layer systems, which suggest trends in the more complex multilayer systems.
\end{abstract}

\section{INTRODUCTION}

It is well known that a variety of fascinating features arise in the study of optical transmission through systems with nonlinear response. For example, optical reciprocity breaks down in nonlinear systems not having mirror symmetry. ${ }^{12}$ This means that transmission through the material in one direction is not necessarily the same as the transmission in the opposite direction. Multistability occurs, allowing a given input to have more than one output, depending on how the input intensity is reached, a hysteretic effect. ${ }^{1-6}$ Also, optical limiting can occur, whereby the output intensity remains at a nearly constant value as the magnitude of the input intensity increases beyond a critical value. These features, combined with the self-induced transparency and chaotic transmission, make nonlinear optical response a topic of wide interest from both applied and fundamental perspectives.

The first mention of optical power limiters was made by Siegman in $1962 .^{7}$ In that work, parametric subharmonic oscillators were suggested as optical limiters with the objective of protecting instrumentation from overload. More recently, Kozlouski $^{8}$ and Edelstein, Wachman, and Tang ${ }^{8,9}$ have discussed optical limiters in the form of parametric oscillators. The first demonstration of passive limiting by selfdefocusing was performed by Leite, Porto, and Damen in 1967. ${ }^{10}$ A number of recent papers have examined optical limiting in semiconductors, ${ }^{11-15}$ organics, ${ }^{16-21}$ fullerenes, ${ }^{22-28}$ and ferroelectric liquid crystals. ${ }^{29}$ For semiconductors the dominant mechanisms are multiphoton absorption and self-defocusing, while for organics and fullerenes reverse saturable absorption dominates. The ferroelectric liquid crystals depend upon electrical linear feedback

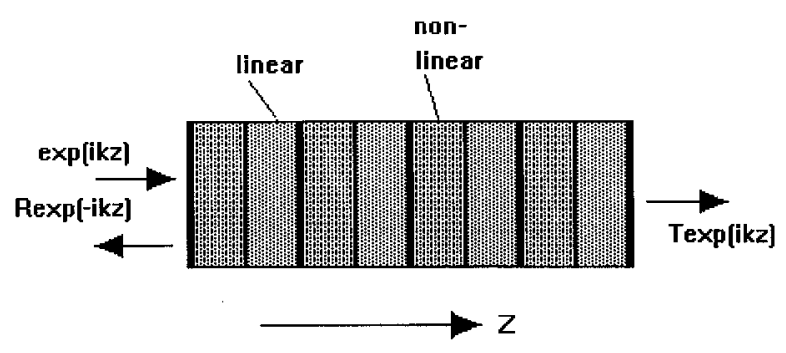

FIG. 1. Model of a periodic superlattice with layers of alternating linear and nonlinear response. to obtain optical limiting behavior. A review of optical limiting mechanisms can be found in Ref. 30.

In this paper, we will take a different approach by modeling materials that have periodic multilayer structures, and examining the optical transmission that occurs when alternate layers exhibit nonlinear response. The nonlinearity, which will be represented by a Kerr nonlinearity, has the effect that the transmission coefficient varies with the inten-
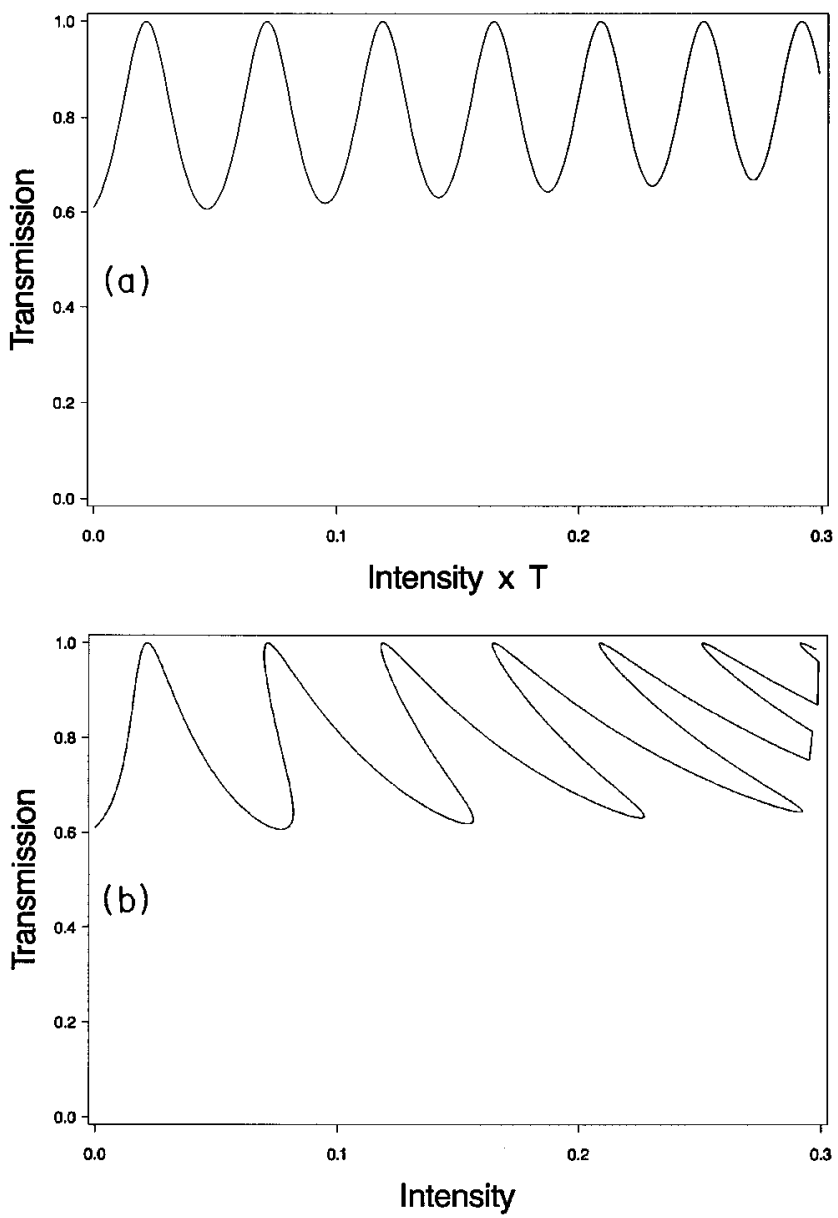

FIG. 2. (a) Transmission vs scaled intensity $\left(\left|E_{0}\right|^{2}=\left|E_{i}\right|^{2}|T|^{2}\right)$ for the system modeled in Fig. 1. The linear dielectric constant $\varepsilon_{L}=2.25$ and the nonlinear dielectric constant $\varepsilon_{\mathrm{NL}}=4.5$, for a layer thickness of $d=\lambda$ (vacuum wavelength) for a ten-bilayer structure. (b) Transmission vs intensity $\left(\left|E_{i}\right|^{2}\right.$ ) for the same parameters as in (a). 


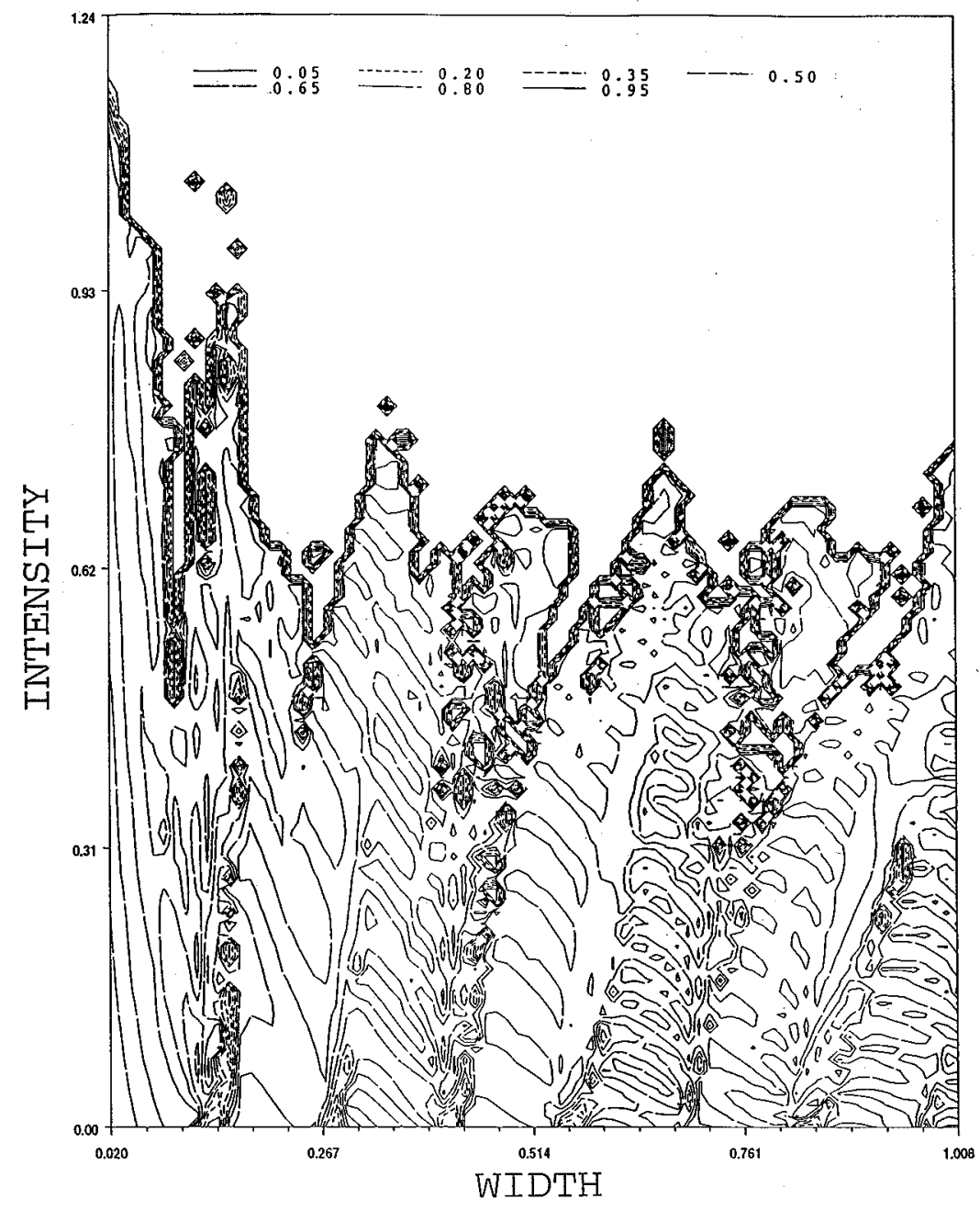

FIG. 3. Contour plot of transmission vs scaled intensity and layer thickness (in units of vacuum wavelength), for a ten-bilayer system with alternating layers with linear and nonlinear response. The linear dielectric constant $\varepsilon_{L}=2.25$ and the nonlinear dielectric constant $\varepsilon_{\mathrm{NL}}=4.5$.

sity of the input signal, as well as with its wavelength. A multilayer structure has previously been explored by Yoo and Alfano ${ }^{31}$ using an approximate scheme to represent nonlinear effects. This work goes beyond their uniform-intensity approximation. We have found that a general feature of these systems is optical limiting for a wide variety of parameters, with parameter-dependent threshold intensities. The threshold intensity is defined to be the minimum intensity for

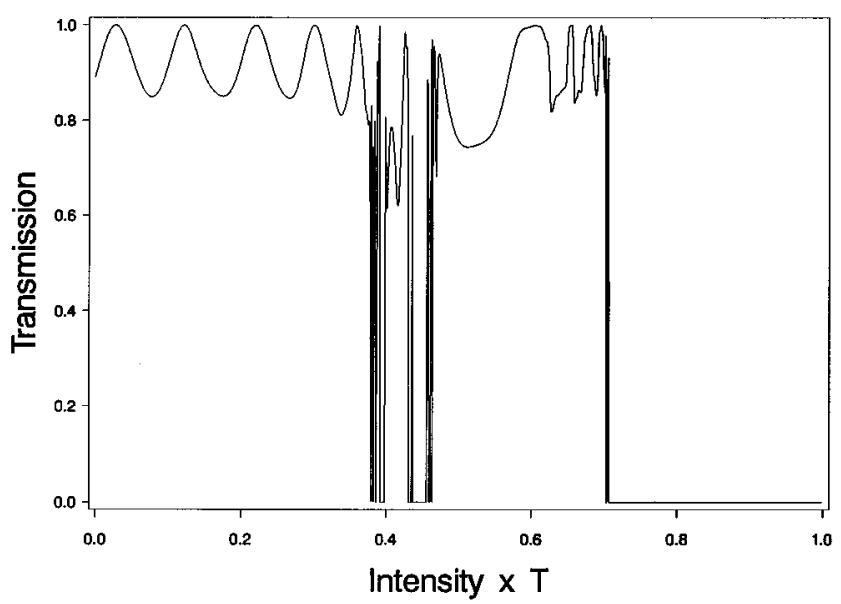

FIG. 4. Cross section of Fig. 3 for layer thickness $d=0.5 \lambda$, where $\lambda$ is the vacuum wavelength. which the output intensity saturates; that is the onset of optical limiting. Understanding the frequency dependence and magnitude of the threshold intensity will be the central aim of this work.

The outline of the paper is as follows. In Sec. II, a mathematical review of the nonlinear formalism for multilayers is given. In Sec. III, the application of the formalism to optical limiting will be presented. In Sec. IV, a survey of results for

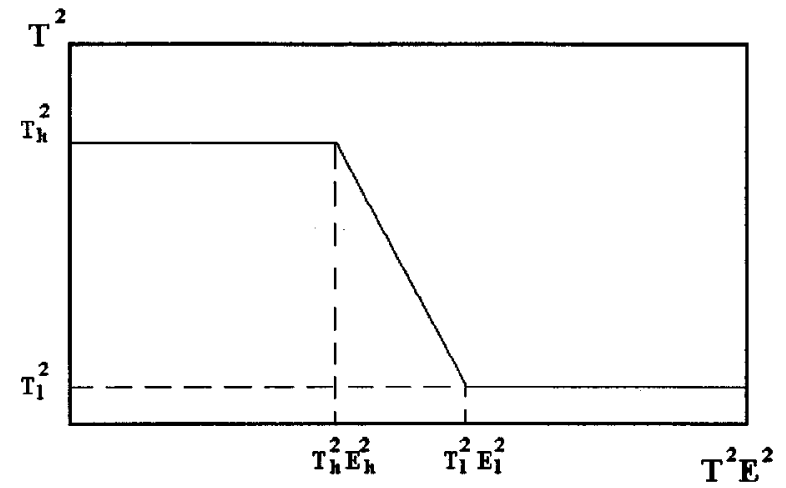

FIG. 5. Model falloff of transmission with input intensity; see text. 


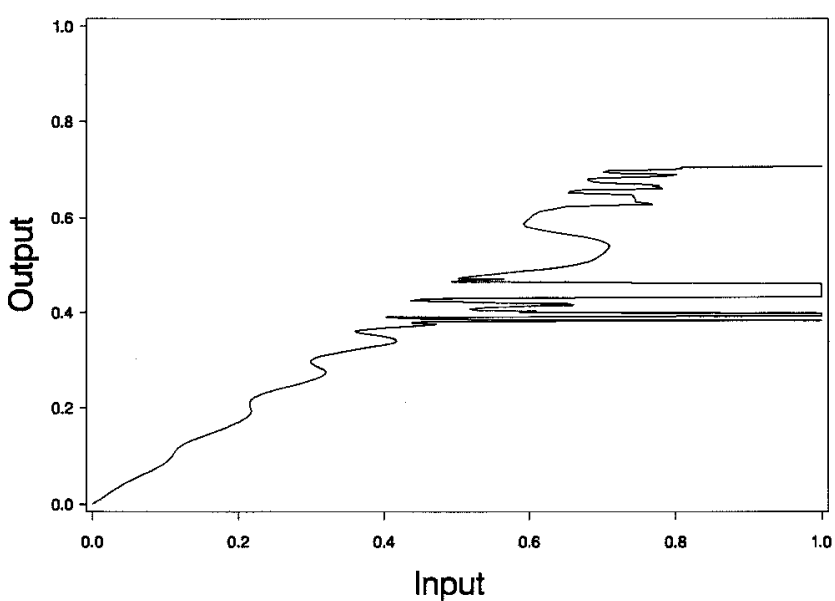

FIG. 6. Output intensity vs input intensity for the parameters in Fig. 4.

a variety of parameters describing optical limiting is given. In Sec. V, a discussion of future work is presented.

\section{BACKGROUND}

We begin with an incident transverse electromagnetic wave in the vacuum, impinging on the multilayer material and propagating in a direction normal to the interfaces. Refer

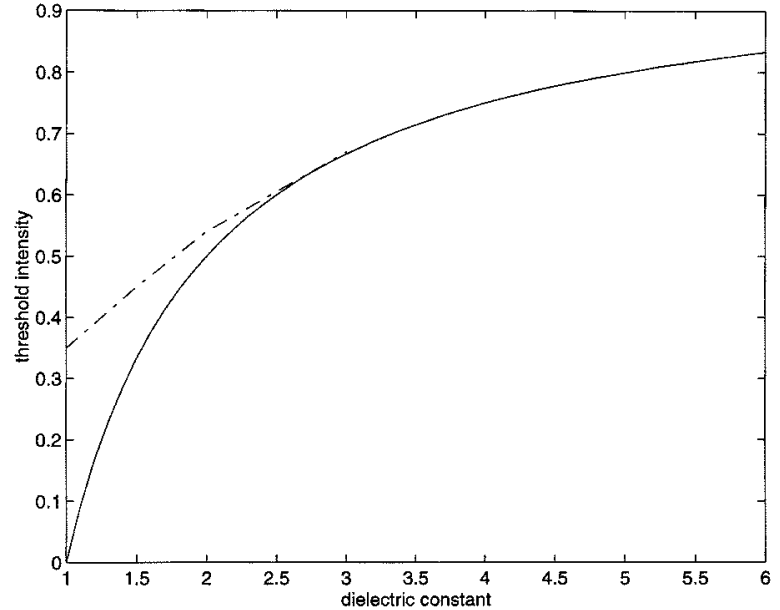

FIG. 7. The threshold intensity for optical limiting in the case of a single-layer structure is shown as a function of the dielectric constant. The solid line curve is for the approximate analytic solution $\left|E_{0}\right|^{2}=1-1 / \varepsilon$. The dot-dash curve displays the numerical results for the full numerical model.

to Fig. 1. The one-dimensional nonlinear equation for the electric field that describes a Kerr nonlinearity is

$$
\frac{d^{2} E}{d z^{2}}+k_{0}^{2} \epsilon\left(1+g|E|^{2}\right) E=0,
$$

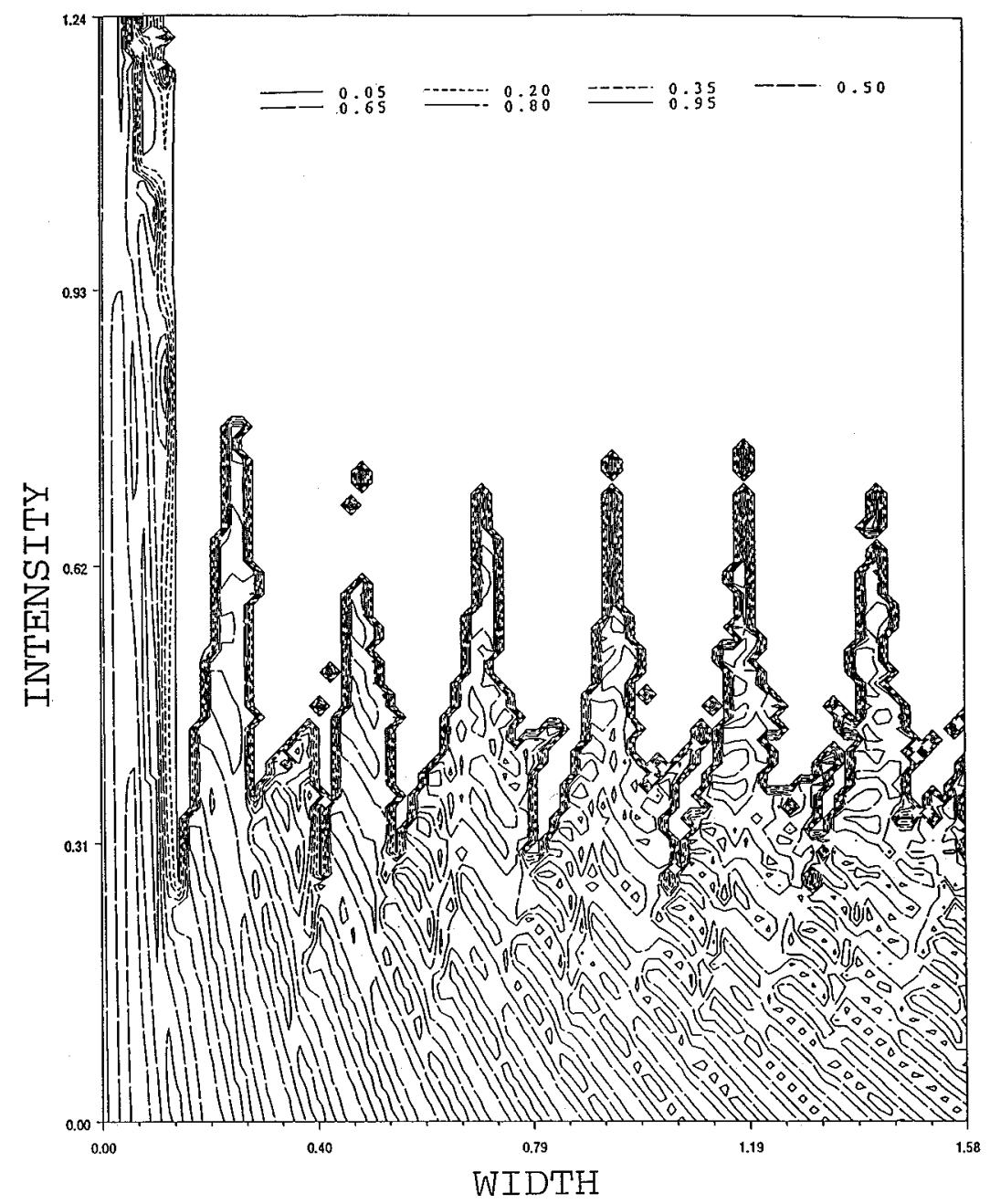

FIG. 8. $|T|^{2}$ vs $\left|E_{0}\right|^{2}$ (scaled intensity) and $d$ for a ten-bilayer system with alternating layers of linear and nonlinear responses both of which have a dielectric constant $\varepsilon=4.5$. 
where $E(z)$ is the position-dependent electric field, $z$ the axis perpendicular to the interfaces, $\omega_{0}$ the frequency of the light, $k_{0}=\omega_{0} / c$ (where $c$ is the velocity of light), $\varepsilon$ is the dielectric function in the basal plane, and the parameter $g$ describes the lowest-order nonlinear coupling. This consists of alternating layers of linear and nonlinear materials. Therefore, the nonlinear parameter $g$ is nonzero in the nonlinear layer and zero in the linear layer. (The selection of $g<0$ in the nonlinear material yields solitons at the bottom of the gap region, while $g>0$ will give solitons at the top of the gap.) Letting $E_{i}$ be the incident field, we define $\xi=E / E_{i}$ and rewrite Eq. (2.1) in the form

$$
\frac{d^{2} \xi}{d z^{2}}+k_{0}^{2} \xi=k_{0}^{2}\left[1-\epsilon\left(1+g|\xi|^{2}\left|E_{i}\right|^{2}\right)\right] \xi .
$$

The boundary conditions, corresponding to the vacuum region, are given by

$$
\begin{gathered}
\xi=\exp \left(i k_{0} z\right)+R \exp \left(-i k_{0} z+i \phi\right), \quad z \leqslant 0, \\
\xi=T \exp \left(i k_{0} z\right), \quad z \geqslant L,
\end{gathered}
$$

where $L$ is the length of the multilayer structure, $R$ is a real number describing the reflectivity, and $T$ is the transmission coefficient. At each interface the continuity of the tangential $E$ and $d E / d z$ is maintained. After scaling, $\Psi=\xi / T$ and discretizing Eq. (2.2), we arrive at

$$
\Psi_{n-1}=2 \psi_{n}-\psi_{n+1}-K^{2} \epsilon\left(1+g\left|E_{i}\right|^{2}|T|^{2}\left|\psi_{n}\right|^{2}\right) \psi_{n} .
$$

Note that $\Psi$ has real and imaginary parts, so that the iteration expressed in Eq. (2.4) represents two equations. The vacuum region boundary conditions corresponding to Eq. (2.4) become

$$
\begin{gathered}
\psi_{n}=R_{0} \exp (i K n)+R_{1} \exp (-i K n+i \delta), \quad n \leqslant 0 \\
\psi_{n}=\exp (i K n+i \phi), \quad n \geqslant \frac{L}{\Delta},
\end{gathered}
$$

where $K=k_{0} \Delta, \Delta$ being the width of the interval in the basic spatial grid. The interlayer boundary conditions are given by

$$
\begin{gathered}
\psi_{n}=\psi_{n+1}, \\
\psi_{n}-\psi_{n-1}=\psi_{n+1}-\psi_{n} .
\end{gathered}
$$

Equations (2.4), (2.5), and (2.6), accompanied by the width of layers in the material, represent the basic model which we are concerned. This formalism readily lends itself to the modeling of absorption by including an imaginary part to the dielectric constant used in Eq. (2.4).

The transmission coefficient is determined as described in Ref. 2. The essentials are as follows. Equation (2.4) is integrated from the output end, with the initial condition $\left|\Psi_{n}\right|=1,\left|E_{i}\right|^{2}|T|^{2}=\left|E_{0}\right|^{2}$, and the initial derivative of the

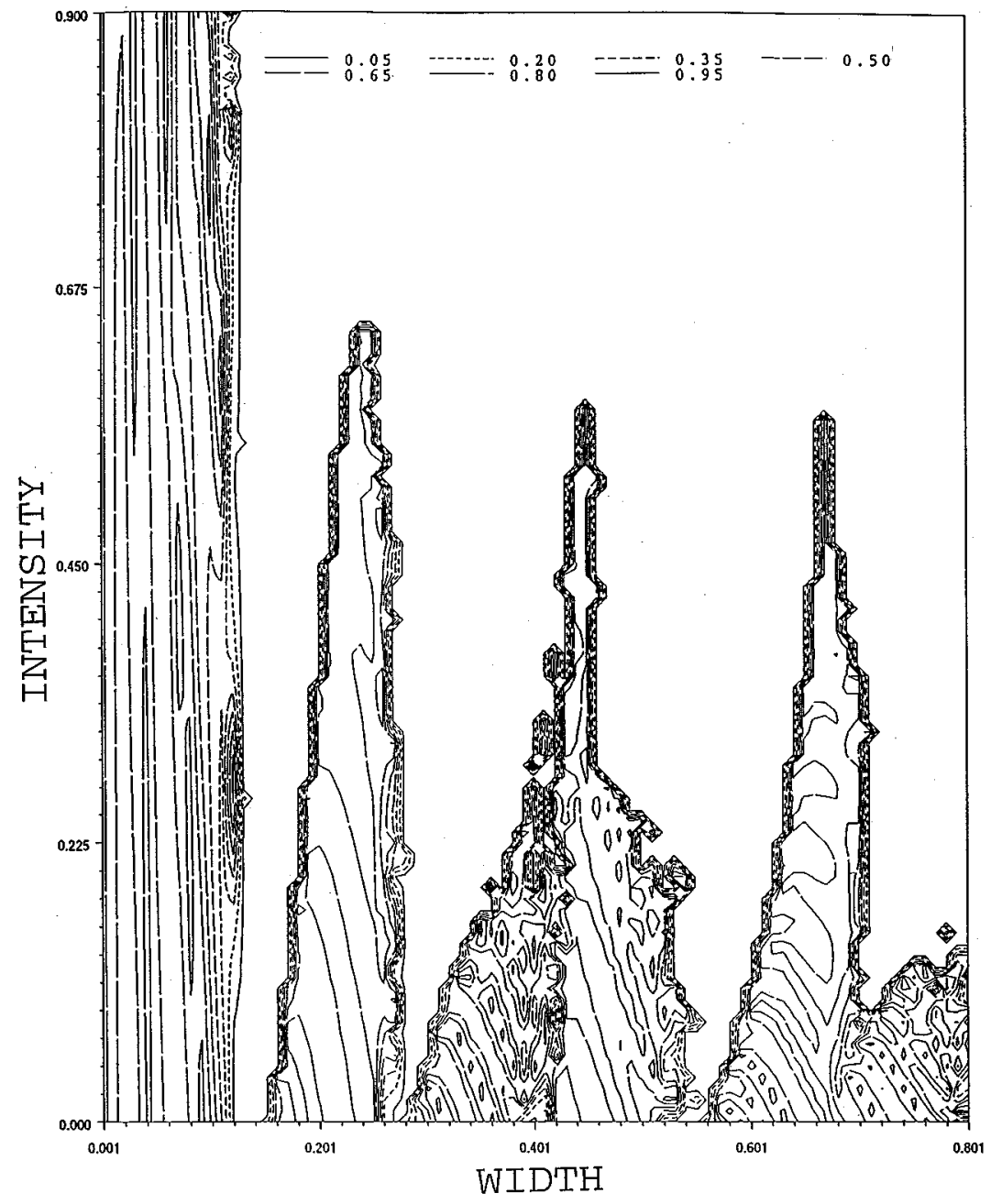

FIG. 9. $|T|^{2}$ vs $\left|E_{0}\right|^{2}$ (scaled intensity) and $d$ for a ten-bilayer system with alternating layers of linear $\left(\varepsilon_{L}=5.0\right)$ and nonlinear $\left(\varepsilon_{\mathrm{NL}}=2.25\right)$ responses. 
intensity with respect to position set equal to zero. At each interface the boundary conditions of Eq. (2.6) are employed. By integrating into the vacuum on the input side, the incident intensity is determined from which the transmission coefficient is calculated [the transmitted intensity has unit magnitude, see Eq. (2.5)]. Since $g$ and $E_{i}$ only appear as the product $g\left|E_{i}\right|^{2}$, we will choose $g= \pm 1$ and only vary the magnitude of $E_{i}$. The resultant information is in the form of $|T|^{2}$ vs $\left|E_{0}\right|^{2}$. It should be noted that this is a one-to-one functional form; for each $\left|E_{i}\right|^{2}$ one $|T|^{2}$ is found. While this format is convenient for viewing the results, it is important to remember that to recover the transmission coefficient as a function of the input intensity, $\left|E_{0}\right|^{2}$ must be divided by $|T|^{2}$. That is, the value of the abscissa must be rescaled by the value of $|T|^{2}$ to recover the multivalued $|T|^{2}$ vs $\left|E_{i}\right|^{2}$. In
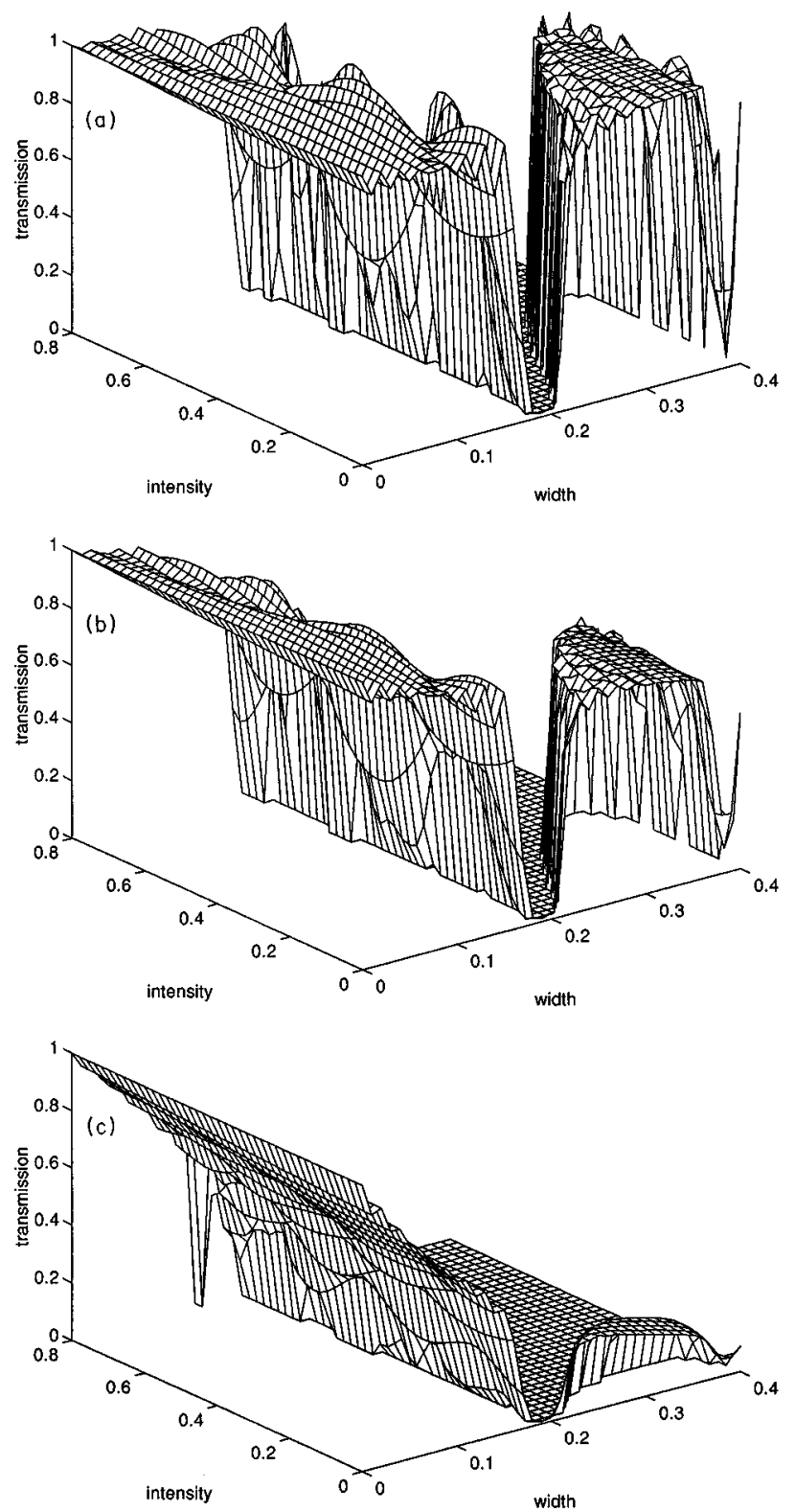

FIG. 10. Transmission vs scaled intensity and layer thickness for a ten-bilayer system having $\varepsilon_{L}=2.25$ and $\varepsilon_{\mathrm{NL}}=1.25$ for (a) the imaginary part of the dielectric functions equaling 0 , (b) the imaginary part of the dielectric functions equaling 0.01 , and (c) the imaginary parts of the dielectric functions equaling 0.05 .
Fig. 2 a comparison of the two methods of display is shown. This more common format suffers from the disadvantage that the value of $\left|E_{i}\right|^{2}$ becomes extremely large for small values of $|T|^{2}$, resulting in the loss of resolution in the interesting regions. In Sec. III we will apply this formalism to the problem of optical limiting.

\section{OPTICAL LIMITING}

What brought our attention to the question of optical limiting was Fig. 3, which is a contour map of $|T|^{2}$ versus $\left|E_{0}\right|^{2}$ and layer width. The layer width is denoted by $d$, and measured in terms of the vacuum wavelength $\lambda_{0}$. This figure, presented in the scaled format, shows a rapid falloff of $|T|^{2}$ as $\left|E_{0}\right|^{2}$ is increased for all layer thicknesses shown (or equivalently for all frequencies). A cross section for $d / \lambda_{0}=0.5$ is given in Fig. 4, highlighting the rapid drop in $|T|^{2}$. The "coastline" profile caught our attention and aroused our curiosity. The similar nature of the threshold to that studied for nonlinearities arising from antiferromagnetic resonances was noted; however, in that case a seemingly chaotic $|T|^{2}$ appears after a comparable threshold. ${ }^{2}$

The difficulty of plotting this in the standard format is that the point at the bottom of the drop in transmission corresponds to an incident intensity, $\left|E_{i}\right|^{2}=\left|E_{0}\right|^{2} /|T|^{2} \gg 10^{12}$. We can, however, extract what is happening in the threshold region through the use of the following approximation. As shown in Fig. 5, we approximate the falloff in a linear fashion, dropping from $\left|T_{h}\right|^{2}$ to $\left|T_{l}\right|^{2}$ in the region $\left|T_{h}\right|^{2}\left|E_{h}\right|^{2}$ to $\left|T_{l}\right|^{2}\left|E_{l}\right|^{2}$. In the limit that $\left|T_{h}\right|^{2}\left|E_{h}\right|^{2}$ approaches $\left|T_{l}\right|^{2}\left|E_{l}\right|^{2}$ (to represent the sharp falloff that is observed), this model leads to

$$
|T(E)|^{2}=\left|T_{h}\right|^{2} \frac{\left|E_{h}\right|^{2}}{|E|^{2}},
$$

so that $|T(E)|^{2}$ is a smooth function of $|E|^{2}$, the input intensity. The output intensity defined by $\left|E_{0}\right|^{2}=|T(E)|^{2}|E|^{2}$ becomes the constant $\left|E_{0}\right|^{2}=\left|T_{h}\right|^{2}\left|E_{h}\right|^{2}$, after substituting for $|T|^{2}$. That is, after the threshold incident intensity $\left|E_{h}\right|^{2}$ is reached, the output intensity becomes a constant. This is an ideal realization of optical limiting.

A more informative way of plotting the results is as $\left|E_{0}\right|^{2}$ vs $\left|E_{i}\right|^{2}$, as shown in Fig. 6. The horizontal line indicates the constant output as a function of input. Technically these lines have a slight slope, which in the example illustrated is 0.0001 .

The origin of the threshold illustrated in Fig. 4 can be understood if we look at the differential equation equivalent to the discrete equation (2.4),

$$
\frac{d^{2} \psi}{d z^{2}}=-k_{0} \epsilon\left(1+\left.g\left|E_{0}\right|^{2} \psi\right|^{2}\right) \psi
$$

At the threshold $|\psi| \gg 1$, and so this equation can be very closely approximated by

$$
\frac{d^{2} \psi}{d z^{2}}=-k_{0} \epsilon\left(g\left|E_{0}\right|^{2}|\psi|^{2}\right) \psi
$$


near the threshold. The solution to this equation can be examined in the two cases $g=+1$ and $g=-1$. In the case $g=-1$, the solutions are of the form

$$
\psi=\frac{1}{\alpha-\beta z}
$$

In this expression $\beta=\sqrt{\left(k_{0} \varepsilon\left|E_{0}\right|^{2} / 2\right)}$, and $\alpha$ is determined by the initial conditions. For $\alpha>0$, the solution clearly exhibits a singularity in the region of interest $(z>0)$, and is found accurately to describe the electric field intensity for values of $\left|E_{0}\right|^{2}$ near the threshold when compared with numerical solutions. For $\alpha<0$, the solution leads to a decaying value of $|\Psi|$, for $z>0$, so that eventually the neglected term in Eq. (3.2) would become significant and the more common oscillatory propagation would occur. The solution which results depends on the initial conditions. When Eq. (3.2) is solved numerically, for a given initial condition such that the part of the solution consistent with the singularity grows, optical limiting occurs.

For $g=+1$, the solutions are of the form

$$
\begin{gathered}
\psi=A \exp (-i B z), \\
\psi=A \exp (i B z),
\end{gathered}
$$

No singularity and, therefore, no optical limiting behavior is seen for this case.

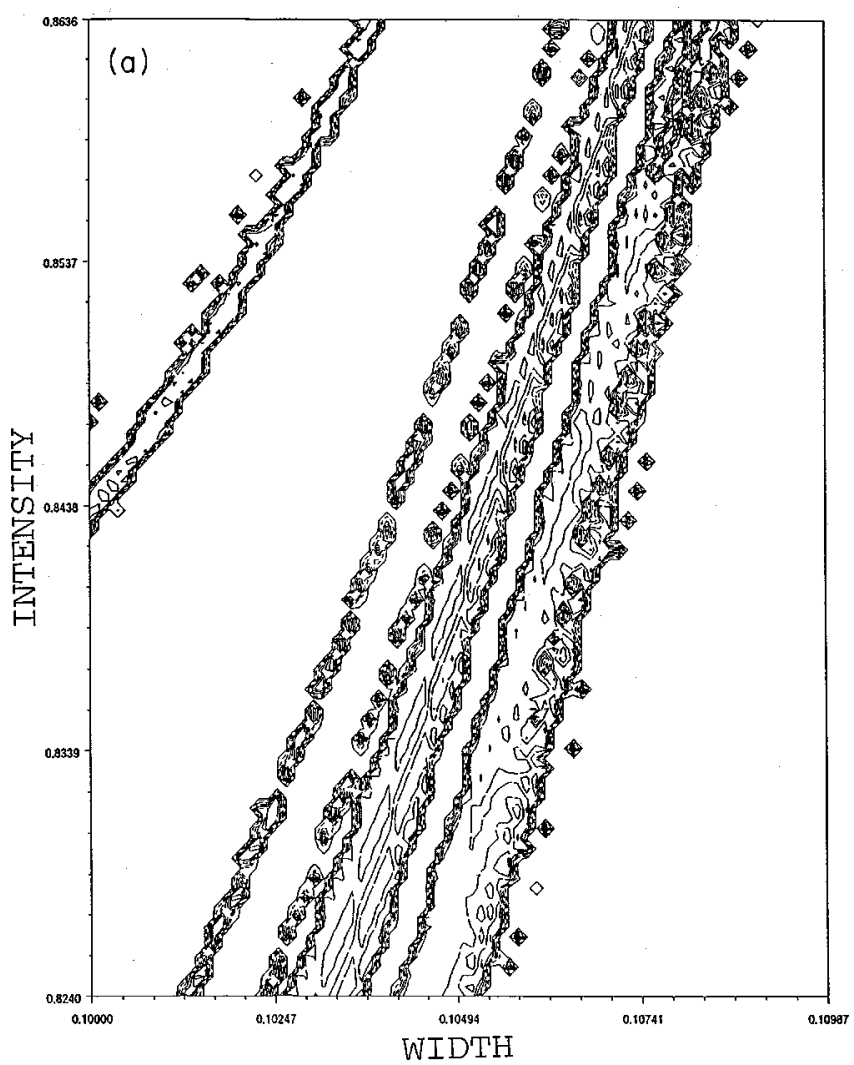

\section{OPTICAL LIMITING-EXAMPLES}

While Fig. 3 is interesting in and of itself, practical application of optical limiters necessitates the ability to control the threshold intensity for a variety of frequencies. A singlelayer system offers an opportunity to examine the characteristics that might be expected in more complex systems. In our discussion, the single layer will always be at least two vacuum wavelengths thick in order to insure that optical limiting will not be masked by inadequate material thickness. [As shown in Sec. III, $\Psi=1 /(\alpha-\beta z)$ in the case of optical limiting. If the material does not include the point $z=\alpha / \beta$, optical limiting will be curtailed.]

In the single-layer case, as in the multilayer case [see Eq. (2.5)], the solution in the vacuum on the transmission side is given by $\Psi=\exp (i k z)$ with $d \Psi / d z=i k \exp (i k z)$. Inside the material, $\Psi$ obeys

$$
\frac{d^{2} \psi}{d z^{2}}=-k^{2} \epsilon\left(1-\left|E_{0}\right|^{2}|\psi|^{2}\right) \psi,
$$

where $g=-1$ has been introduced explicitly (recall that $g=+1$ does not yield optical limiting). The boundary conditions require continuity of $\Psi$ and $d \Psi / d z$. Since in the vacuum $|\Psi|^{2}=1$, continuity would imply that $|\Psi|^{2} \cong 1$ in the material, near the interface. The numerical results can be modeled analytically by making this substitution in Eq. (4.1). This results in the approximate equation

$$
\frac{d^{2} \psi}{d z^{2}}=-k^{2} \epsilon\left(1-\left|E_{0}\right|^{2}\right) \psi
$$

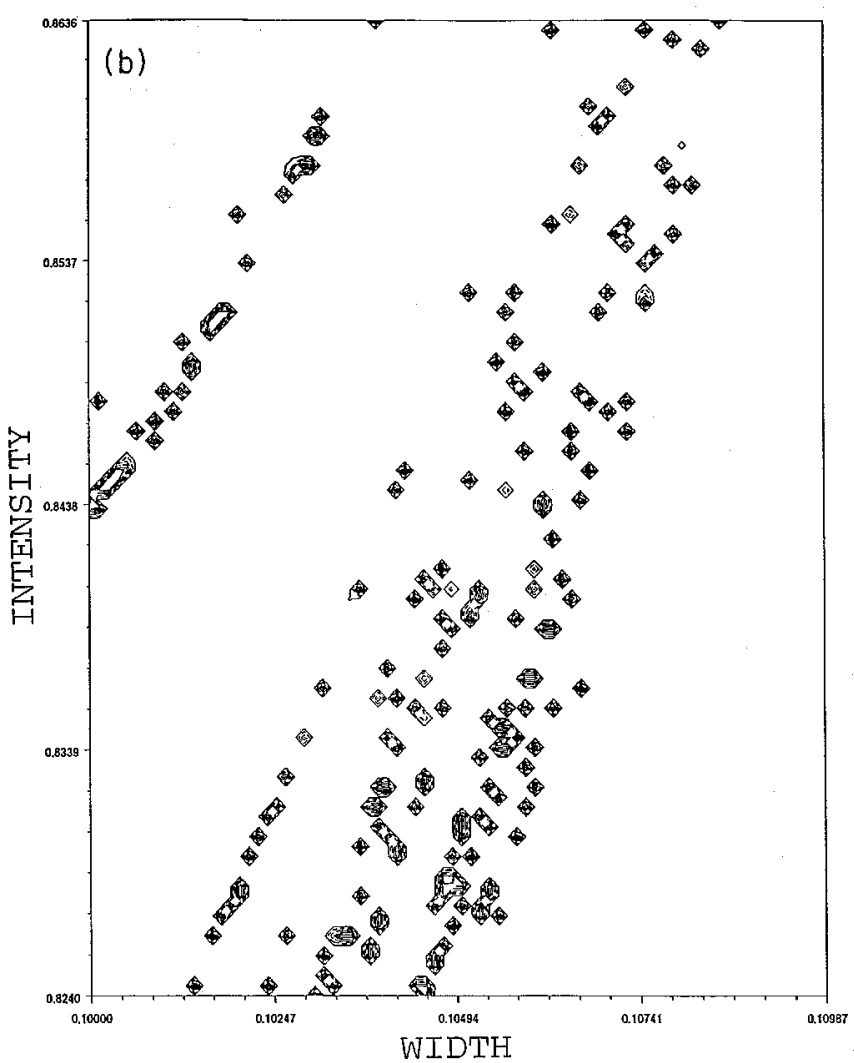

FIG. 11. (a) Same as Fig. 3, except that a smaller region in parameter space is chosen. (b) Same as (a), but with 20 bilayers rather than ten bilayers. Legends are the same as in Fig. 3. 


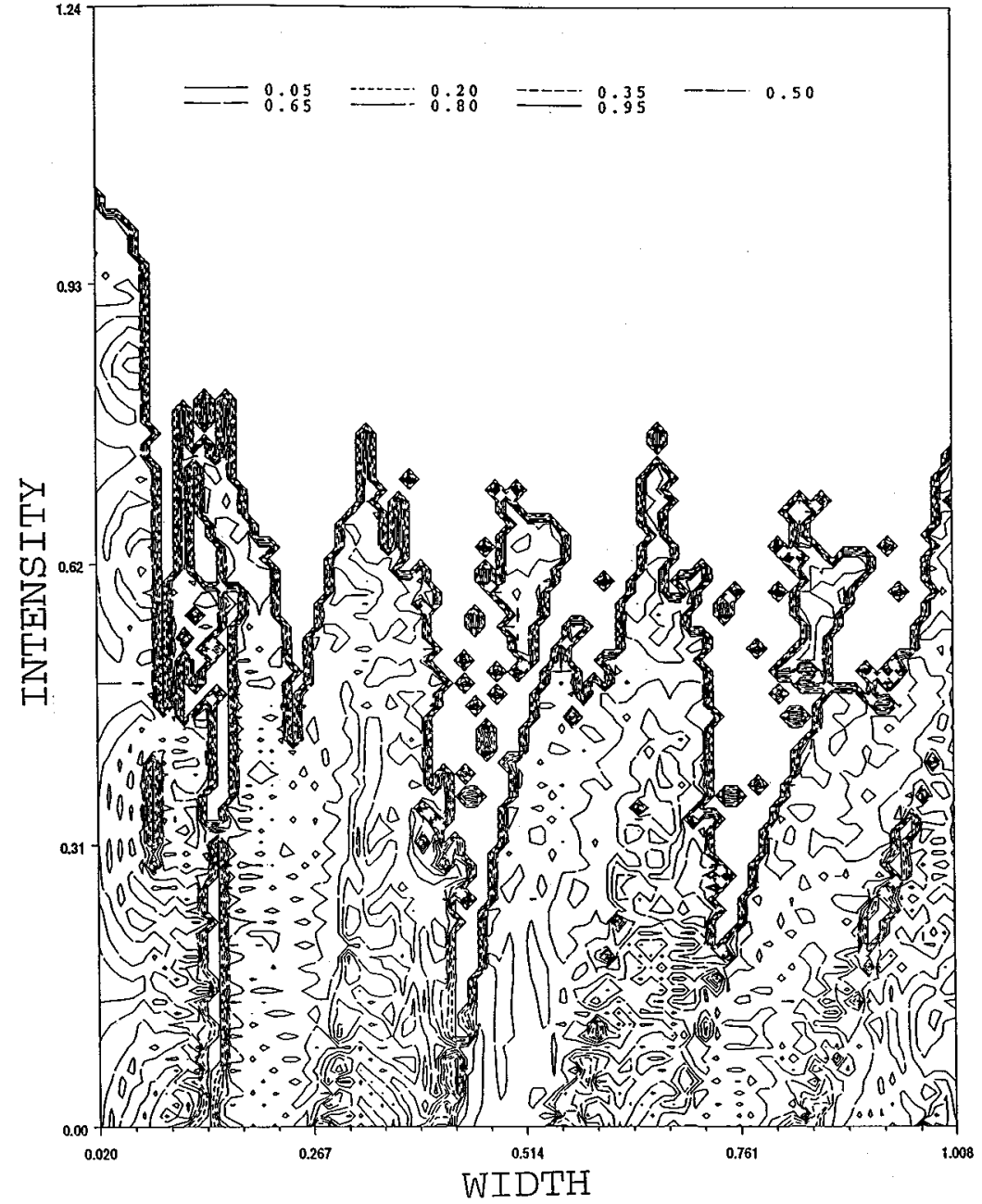

FIG. 12. Same as Fig. 3, but with 80 bilayers rather than ten bilayers. which has solutions

$$
\begin{aligned}
\psi= & A \exp \left[i k z \sqrt{\left(1-\left|E_{0}\right|^{2}\right) \epsilon}\right] \\
& +B \exp \left[-i k z \sqrt{\left(1-\left|E_{0}\right|^{2}\right) \epsilon}\right] .
\end{aligned}
$$

On matching boundary conditions with the vacuum side, and defining $S=\sqrt{\left[\left(1-\left|E_{0}\right|^{2}\right) \epsilon\right]}$, we find

$$
A=\frac{1}{2}\left(1+\frac{1}{S}\right) \exp [i k L(1-S)]
$$

and

$$
B=\frac{1}{2}\left(1-\frac{1}{S}\right) \exp [i k L(1+S)]
$$

Note that when

$$
\epsilon\left(1-\left|E_{0}\right|^{2}\right)=1,
$$

$A=1$ and $B=0$ yield a harmonic solution for which $|\Psi|^{2}=1$, and no singularities are found. This is the boundary between the optical limiting solutions and the regular solutions in our analytical model.

If $\varepsilon\left(1-\left|E_{0}\right|^{2}\right)>1$, then $1 / 2<|A|<1$ and $1 / 2>|B|>0$. This results in solutions for which $|\Psi|^{2} \leqslant 1$ and a nonsingular behavior evolves. If $\varepsilon\left(1-|E|^{2}\right)<1$, then $|A|>1$, $|B|>0$, and $|\Psi|^{2} \geqslant 1$; a monotonic growing solution evolves and the singular behavior described in Sec. III develops. No dependence of the threshold intensity on the vacuum wavelength is expected for a single-layer material.

These predictions can be compared with the numerical results. Numerically, it is found that $\left|E_{0}\right|^{2} \leqslant 1-1 / \varepsilon$ yields regular transmission and $\left|E_{0}\right|^{2}>1-1 / \varepsilon$ yields optical limiting for nearly all values of $\varepsilon>1$. The exception to this rule is the region of small $\varepsilon<2.0$, where $\left|E_{0}\right|^{2}<0.5$. In this region, the nonlinear term in Eq. (4.1) grows as the wave proceeds into the material, but is dominated by the linear term. In the case of $\varepsilon=2.0$, an $\left|E_{0}\right|^{2}>0.54$ is required as opposed to the predicted value of 0.50 , found from Eq. (4.6). At smaller values of $\varepsilon$, the required $\left|E_{0}\right|^{2}$ remains above the prediction with $\left|E_{0}\right|^{2}=0.35$ for $\varepsilon=1$; Eq. (4.6) would indicate that all values of $\left|E_{0}\right|^{2}>0$ would yield optical limiting. These results are displayed in Fig. 7. No dependence on the vacuum wavelength is seen numerically.

What is seen from this study of single-layer materials is that, contrary to the naive expectation that a larger $\varepsilon$ would yield a smaller required $\left|E_{0}\right|^{2}$, a smaller $\varepsilon$ will have the smaller threshold $\left|E_{0}\right|^{2}$. In the remaining part of this section a numerical survey of multilayer systems is presented.

To take advantage of multilayer systems for optical limiting, a system is needed which has more flexibility than the frequency-independent single-layer system but not necessarily having the complexity of the system illustrated in Fig. 3. A survey of systems with varying dielectric functions indi- 
cates that the sharp drop in transmission as a function of $\left|E_{0}\right|^{2}$, the signature of optical limiting, is a common occurrence, happening in all systems studied for values of $\left|E_{0}\right|^{2}<1$. This value of $\left|E_{0}\right|^{2}$ might have been expected from the formula for single-layer systems [Eq. (4.6)]. Furthermore, most multilayer systems had a transmission versus $\left|E_{0}\right|^{2}$ and $d$ (see Fig. 3), which was of intermediate complexity.

Figure 8 shows $|T|^{2}$ vs $\left|E_{0}\right|^{2}$ and $d$ for a ten-bilayer system with alternating layers of linear and nonlinear response, both of which have $\varepsilon=4.5$. In the linear case, this system is a single-layer system so that no gaps in the transmission are seen. The single-layer threshold of $\left|E_{0}\right|^{2}=0.78$ is not relevant because the multiple layers have influence through the periodicity of the nonlinearity. This type of contour occurs whenever the two dielectric constants are equal. Note that the layer thickness in Fig. 8 has a wider range than that of Fig. 3.

Figure 9 illustrates a multilayer system with a band structure, having stopgaps and transmission bands. In this case, the linear-response layer has $\varepsilon=5.0$, and the layer with nonlinear response has $\varepsilon=2.25$. This figure shows that the threshold value of $\left|E_{0}\right|^{2}$ can be made arbitrarily small at frequencies near the band edges. This result is typical of the systems surveyed.

One of the concerns regarding the applicability of multilayer structures for optical power limiting is the effect that absorption would have. To examine this concern we studied the ten-bilayer system having $\varepsilon=2.25$ (linear) and $\varepsilon=1.25$ (nonlinear), which has intensity-frequency regions with nearly constant transmission of nearly $100 \%$ (see Fig. 10). The effect of absorption is modeled with an imaginary part added to the dielectric constants. When an imaginary part equal to 0.01 is added to both dielectric constants, the plateau region drops to a transmission of about $90 \%$. At the same time, the threshold intensity decreases by about $10 \%$. When the imaginary part is 0.05 , the plateau transmission value is $25 \%$, and occupies a significantly smaller region in intensity-frequency space. The threshold is not as sharp as in the nonabsorptive case. A plot of output versus input shows a more gradual approach to constant output. The slope of the "constant" output region is 0.001 as opposed to 0.0001 for the nonabsorptive case. It should be noted that small variations $(\sim 5 \%)$ in individual layer thickness has an insignificant effect on the optical limiting properties (see Ref. 32).

The "islands" which appear in contour maps, such as Fig. 3 , also have some practical applications. These structures are very sharp transmission resonances as a function of intensity and wavelength. We were interested in determining how these structures changed as the superlattice increased in length, keeping the layer thickness constant. This was ac- complished by zooming in on a region of Fig. 3, a ten-bilayer system, and examining the same region for a 20-bilayer system. The results are displayed in Figs. 11(a) and 11(b). As the number of layers are increased, the continuous structures break into islands, while nearly all of the islands, from the ten-bilayer structure, disappear. This is to be compared with what happens in the full region of Fig. 3 when an 80-bilayer structure is modeled in Fig. 12. In this case the "gulfs" penetrate further "inland," and many of the islands disappear. It should be noted that these are nonlinear effects; doubling the length of superlattice does not imply that the transmission should be squared, as would be the case in a linear system. Such a result would yield either perfect transmission or zero transmission in the limit of an infinite superlattice.

\section{CONCLUSIONS}

We have surveyed a number of models of multilayer systems with the intent of determining the usefulness of such systems as optical power limiters. Ideally, an optical limiter would have an output intensity equal to the input intensity (perfectly transmitting) and beyond a certain threshold the output would be constant. It would be most advantageous if the threshold intensity could be made frequency dependent. We have modeled systems that have nearly ideal characteristics. For the case considered in Sec. IV, with $\varepsilon=2.25$ and 1.25 , nearly perfectly transmitting plateaus are seen. In certain regions of $d$, which is equivalent to frequency, the threshold rises monotonically from essentially zero, allowing the layer thickness to be determined by the frequency of radiation and the desired maximum output intensity.

Experimental tests of our model are needed to determine the viability of devices built with these structures. As noted earlier, defects and absorption, within limits, should not significantly affect the optical limiting properties of the systems. The importance of the approximations used in this model (no power to higher harmonics, no effects of lattice mismatches at interfaces, a one-dimensional system, and only a Kerr nonlinearity) must be tested. Materials that can be fabricated into layered systems, with appropriate dielectric constants, must be determined. The frequency range will be dependent on the layer thickness.

Should these experiments verify our model's predictions, these multilayer optical limiters would prove useful in protecting optical devices from harmful overloads. At the same time, the nonlinear nature of these devices, with the inherent nonreciprocity of transmission, suggests that layered systems could be fabricated which would allow intense radiation to pass in one direction but not in the other (at a given frequency). We hope that this work encourages experimentation in this area.
${ }^{1}$ Wei Chen and D. L. Mills, Phys. Rev. Lett. 58, 160 (1987); Phys. Rev. B 35, 524 (1987); 36, 6269 (1987).

${ }^{2}$ L. Kahn, N. S. Almeida, and D. L. Mills, Phys. Rev. B 37, 8072 (1988).

${ }^{3}$ D. L. Mills and S. E. Trullinger, Phys. Rev. B 36, 947 (1987).

${ }^{4}$ L. M. Kahn, K. Huang, and D. L. Mills, Phys. Rev. B 39, 12449 (1989)
${ }^{5}$ N. S. Almeida and D. L. Mills, Phys. Rev. B 36, 2015 (1987).

${ }^{6}$ F. Delyon, Y. E. Levy, and B. Souillard, Phys. Rev. Lett. 57, 2010 (1987).

${ }^{7}$ A. E. Siegman, Appl. Opt. 1, 739 (1962).

${ }^{8}$ W. Kozlouski, Opt. Lett. 14, 66 (1989).

${ }^{9}$ D. C. Edelstein, E. S. Wachman, and C. L. Tang, Appl. Phys. Lett. 54, 1728 (1989). 
${ }^{10}$ R. C. C. Leite, S. P. S. Porto, and T. C. Damen, Appl. Phys. Lett. 10, 100 (1967).

${ }^{11}$ J. M. Ralston and K. R. Chang, Appl. Phys. Lett. 15, 164 (1969).

${ }^{12}$ J. E. Geusic, S. Singh, D. W. Tipping, and T. C. Rich, Phys. Rev. Lett. 19, 1126 (1969).

${ }^{13}$ E. W. Van Stryland, Y. Y. Wu, D. J. Hagan, M. J. Stoileau, and K. Mansour, J. Opt. Soc. Am. B 5, 1981 (1988).

${ }^{14}$ D. J. Hagan, E. W. Van Stryland, M. J. Sioleau, and Y. Y. Wu, Opt. Lett. 13, 315 (1988)

${ }^{15}$ W. Ji, A. K. Kukaswadia, Z. C. Feng, and S. H. Tang, J. Appl. Phys. 75, 3340 (1994).

${ }^{16}$ J. S. Shirk, M. E. Boyle, J. D. Adkins, G. S. R. Pong, S. R. Flom, and J. R. Lindle, in Proceedings of the Conference on Lasers and Electro-Optics, Anaheim, CA, 1994 (IEEE, Piscataway, NJ, 1994).

${ }^{17}$ Q. W. Song, C. Zhang, R. Gross, and R. Birge, Opt. Lett. 18, 775 (1993).

${ }^{18}$ P. D. Fuqua, K. Mansour, D. Alvarez, Jr., S. Marder, J. W. Perry, and B. S. Dunn, Proc. SPIE 1758, 499 (1992).

${ }^{19}$ L. V. Natrajan, R. L. Sutherland, M. G. Schmitt, M. C. Brandt, D. G. McLean, P. A. Fleitz, T. J. Bunning, and R. L. Crane, Proc. SPIE Int. Soc. Opt. Eng. (USA) 1853, 99 (1993).

${ }^{20}$ K. Mansour, D. Alvarez, K. J. Perry, I. Choong, S. R. Marder, and J. W. Perry, Proc. SPIE Int. Soc. Opt. Eng. (USA) 1853, 132 (1993).
${ }^{21}$ T. F. Boggess, G. R. Allen, S. J. Rychnovsky, D. R. Labergerie, C. H. Venzke, A. L. Smirl, L. W. Tutt, A. R. Kost, S. W. McCahon, and M. B. Klein, Opt. Eng. Bellingham (USA) 32, 1063 (1993).

${ }^{22}$ S. R. Misra, H. S. Rawat, M. P. Joshi, and M. C. Mahandale, J. Phys. B 27, L157 (1994).

${ }^{23}$ B. L. Justus, Z. H. Kafafi, and A. L. Huston, Opt. Lett. 18, 1603 (1993).

${ }^{24}$ A. Kost, L. Tutt, M. B. Klein, T. K. Dougherty, and W. E. Elias, Opt. Lett. 18, 334 (1993).

${ }^{25}$ C. Li, Y. Wang, F. Guo, R. Wang, and L. Zhang, Acta Phys. Sin. 42, 1236 (1993).

${ }^{26}$ G. Qihuang, Y. Shaochen, S. Yuxing, X. Zongju, Z. Yingua, Z. Jin, X. Zhou, Z. Gu, and D. Qiang, J. Infrared Millimeter Waves 12, 110 (1993).

${ }^{27}$ M. P. Joshi, S. R. Mishra, H. S. Rawat, S. C. Mahandale, and K. C. Rustagi, Appl. Phys. Lett. 62, 1763 (1993).

${ }^{28}$ S. Yang, Q. Gong, Y. Sun, Z. Xia, Y. Zou, L. Fei, X. Zhou, D. Qiang, Z. Gu, Y. Wu, and Y. Sun, Acta Opt. Sin. 13, 289 (1993).

${ }^{29}$ A. Tagawa, H. Moritake, M. Ozaki, K. Yoshino, Electron. Commun. Jpn. II 75, 33 (1992).

${ }^{30}$ L. W. Tutt and T. F. Boggess, Prog. Quantum Electron. 17, 299 (1993).

${ }^{31}$ K. M. Yoo and R. R. Alfano, Opt. Lett. 16, 1823 (1991).

${ }^{32}$ L. M. Kahn (unpublished). 